\title{
Scattering Amplitudes from Intersection Theory
}

\author{
Sebastian Mizera* \\ Perimeter Institute for Theoretical Physics, Waterloo, Ontario N2L 2Y5, Canada \\ and Department of Physics and Astronomy, University of Waterloo, Waterloo, Ontario N2L 3G1, Canada
}

(Received 14 November 2017; revised manuscript received 28 February 2018; published 6 April 2018)

We use Picard-Lefschetz theory to prove a new formula for intersection numbers of twisted cocycles associated with a given arrangement of hyperplanes. In a special case when this arrangement produces the moduli space of punctured Riemann spheres, intersection numbers become tree-level scattering amplitudes of quantum field theories in the Cachazo-He-Yuan formulation.

DOI: 10.1103/PhysRevLett.120.141602

Introduction.-Over the past years, study of scattering amplitudes revealed many unexpected connections to geometric structures [1-4], allowing us to understand physical properties of quantum field theories - such as locality or unitarity - from a different perspective. At the same time, they equip us with new mathematical tools that vastly simplify practical calculations. In this work we unravel another connection to a branch of mathematics called intersection theory [5-8].

It has recently transpired that intersection theory plays an important role in string theory amplitudes, where, in particular, it provides a geometric interpretation of the Kawai-Lewellen-Tye (KLT) relations between open and closed string amplitudes, or-in the field-theory limitYang-Mills and Einstein gravity amplitudes [9,10]. Here, we show that analogous structures appear directly in scattering amplitudes of ordinary quantum field theories. We find that they can be understood as intersection numbers of the so-called twisted cocycles [6-8], which are certain families of differential forms.

It is instructive to start with an explicit example straightaway. Let us consider $\mathbb{C P}^{2}$ with inhomogeneous coordinates $(x, y)$, dissected by six hyperplanes defined through linear equations $\left\{f_{i}=0\right\}$. We can easily visualize the real section of this space with a concrete choice of hyperplanes, for instance,

$$
\begin{aligned}
& f_{1}=x, \\
& f_{2}=y, \\
& f_{3}=1-x, \\
& f_{4}=1-x / 4-y, \\
& f_{5}=1 / 4+x-y, \\
& f_{6}=5 / 4-x+2 y .
\end{aligned}
$$

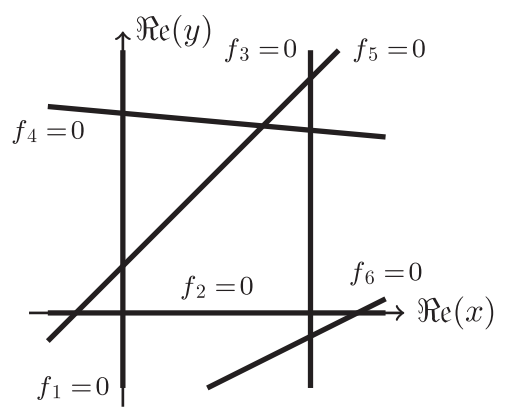

The space of our interest is the original manifold with these hyperplanes removed:

$$
X=\mathbb{C P}^{2} \backslash \bigcup_{i=1}^{6}\left\{f_{i}=0\right\} .
$$

Associated to it, we can define a differential 1-form $\omega$, called the twist, with logarithmic singularities along $f$ 's:

$$
\begin{aligned}
\omega= & \sum_{i=1}^{6} \alpha_{i} d \log f_{i}=\underbrace{\left(\frac{\alpha_{1}}{x}+\frac{\alpha_{3}}{-1+x}+\frac{\alpha_{4}}{-4+x+4 y}+\frac{\alpha_{5}}{1 / 4+x-y}+\frac{\alpha_{6}}{-5 / 4+x-2 y}\right)}_{\omega_{x}} d x \\
& +\underbrace{\left(\frac{\alpha_{2}}{y}+\frac{\alpha_{4}}{-1+x / 4+y}+\frac{\alpha_{5}}{-1 / 4-x+y}+\frac{\alpha_{6}}{5 / 8-x / 2+y}\right)}_{\omega_{y}} d y,
\end{aligned}
$$

Published by the American Physical Society under the terms of the Creative Commons Attribution 4.0 International license. Further distribution of this work must maintain attribution to the author(s) and the published article's title, journal citation, and DOI. Funded by SCOAP ${ }^{3}$. where $\alpha$ 's are constant coefficients adding up to zero. The twist 1-form fully characterizes the space $X$.

On this space we can introduce two differential forms, $\varphi_{L}$ and $\varphi_{R}$. We choose them in such a way that they have 
logarithmic singularities on three of the hyperplanes defined above. For instance, we can take

$\varphi_{L}=d \log \frac{f_{2}}{f_{3}} \wedge d \log \frac{f_{3}}{f_{5}}=\frac{5 d x \wedge d y}{y(1-x)(1+4 x-4 y)}$,

$\varphi_{R}=d \log \frac{f_{2}}{f_{3}} \wedge d \log \frac{f_{3}}{f_{6}}=\frac{d x \wedge d y}{y(1-x)(5-4 x+8 y)}$.

These objects are examples of twisted cocycles, which, roughly speaking, are differential forms on $X$ defined up to equivalence classes $\varphi \sim \varphi+\omega \wedge \xi$ for any $d \log$ form $\xi$. One can define an invariant pairing called the intersection number [11]. Its standard definition [6,7] reads

$$
\left\langle\varphi_{L}, \varphi_{R}\right\rangle_{\omega}=\frac{1}{(2 \pi i)^{2}} \int_{X} l_{\omega}\left(\varphi_{L}\right) \wedge \varphi_{R},
$$

where the map $l_{\omega}$ turns $\varphi_{L}$ into its compactly supported version, i.e., one that vanishes in a small neighbourhood of the hyperplanes $\left\{f_{i}=0\right\}$. Note that the integrand would vanish if it was not for this map. Here we also remark that $\left\langle\varphi_{L}\right|$ and $\left|\varphi_{R}\right\rangle$ belong to different cohomologies, as will be discussed in the following section. A result of this calculation reveals a combinatorial formula [12]:

$$
\left\langle\varphi_{L}, \varphi_{R}\right\rangle_{\omega}= \pm \sum_{\left\{f_{i}, f_{j}\right\} \in L, R} \frac{1}{\alpha_{i} \alpha_{j}}=\frac{1}{\alpha_{2} \alpha_{3}} .
$$

We review the meaning of the map $t_{\omega}$ in Ref. [13], which also illustrates how factors of $\alpha_{i}$ arise in the denominators. The above result is a sum over all intersection vertices of the hyperplanes that are associated to both $\varphi_{L}$ and $\varphi_{R}$. In our example, we have $L=\left(f_{2}, f_{3}, f_{5}\right)$ and $R=\left(f_{2}, f_{3}, f_{6}\right)$, which intersect at a single point $\left\{f_{2}=0\right\} \cap\left\{f_{3}=0\right\}$, and we inserted the correct sign [12]. An important feature of the above formula is that it is completely independent of the precise positions of the hyperplanes, as long as their arrangement is generic; i.e., no three $f$ 's intersect at a single point.

In this Letter we propose an alternative formula for computing intersection numbers as an integral localizing on the points $\left(x^{*}, y^{*}\right)$ at which $\omega$ vanishes:

$$
\begin{aligned}
\left\langle\varphi_{L}, \varphi_{R}\right\rangle_{\omega} & =\int d x d y \delta\left(\omega_{x}\right) \delta\left(\omega_{y}\right) \hat{\varphi}_{L} \hat{\varphi}_{R} \\
& =\left.\sum_{\left(x^{*}, y^{*}\right)} \operatorname{det}^{-1}\left[\begin{array}{cc}
\frac{\partial \omega_{x}}{\partial x} & \frac{\partial \omega_{x}}{\partial y} \\
\frac{\partial \omega_{y}}{\partial x} & \frac{\partial \omega_{y}}{\partial y}
\end{array}\right] \hat{\varphi}_{L} \hat{\varphi}_{R}\right|_{(x, y)=\left(x^{*}, y^{*}\right)},
\end{aligned}
$$

where we used $\varphi=\hat{\varphi} d x \wedge d y$. Here, $\delta$ functions should be understood as multidimensional residue prescriptions around the zeros of $\omega$. Remarkably, this formula evaluates to the rational function of $\alpha$ 's [Eq. (6)] for any choice of $\varphi_{L}$ and $\varphi_{R}$, and does so in a highly nontrivial manner.
Readers familiar with scattering amplitudes literature will notice a resemblance of Eq. (7) to the Cachazo-HeYuan (CHY) formulas [32,33]. This is not a coincidence. In fact, $\mathrm{CHY}$ formalism uses a particular, singular, arrangement of hyperplanes, for example,

$$
\begin{aligned}
\{x=0\} & \cup\{y=0\} \cup\{1-x=0\} \\
& \cup\{1-y=0\} \cup\{x-y=0\},
\end{aligned}
$$

with the last hyperplane at infinity, such that the resulting space $X$ is the moduli space of punctured Riemann spheres, in this case $X=\mathcal{M}_{0,5}$. Equation (6) can no longer be used directly, as the arrangement of hyperplanes is not generic. Nevertheless, the new formula Eq. (7) is still valid. Let us see how this comes about.

We can organize the coefficients of a particular arrangement into a matrix:

$$
\begin{aligned}
& \begin{array}{llllll}
f_{1} & f_{2} & f_{3} & f_{4} & f_{5} & f_{6}
\end{array} \\
& C=\left[\begin{array}{cccccc}
0 & 0 & 1 & 1 & \frac{\varepsilon}{4} & \frac{4+\varepsilon}{4} \\
1 & 0 & -1 & -\frac{\varepsilon}{4} & 1 & -\varepsilon \\
0 & 1 & 0 & -1 & -1 & 2 \varepsilon
\end{array}\right] \begin{array}{l}
1 \\
x \\
y
\end{array},
\end{aligned}
$$

giving $f_{i}=c_{1 i}+c_{2 i} x+c_{3 i} y$. We set it up such that $\varepsilon=1$ yields the original arrangement, which deforms into the singular one as $\varepsilon \rightarrow 0$. A sign of singularity is that several $3 \times 3$ minors of $C$ vanish in this limit. Keeping parameters $\alpha$ constant for example, with $\alpha_{i}>0$ for $i=1,2, \ldots, 5$, the hyperplanes and zeros of $\omega$ behave as follows:

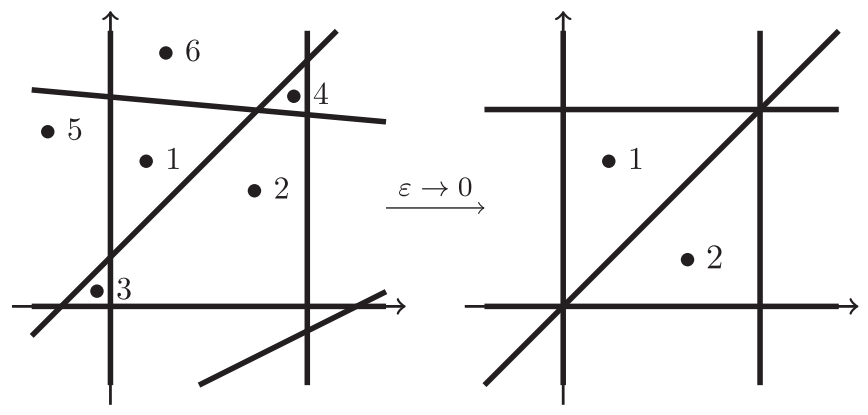

The hyperplane $\left\{f_{6}=0\right\}$ moved to infinity. Out of the six zeros of $\omega$, only 1 and 2 survive at finite positions. Both 3 and 4 get trapped between three hyperplanes and eventually cease to be zeros of $\omega$ since $(0,0)$ and $(1,1)$ are not a part of the manifold $X$. Similarly, 5 and 6 shoot off to infinity. This can be easily verified from the explicit form of the twist in the strict $\varepsilon \rightarrow 0$ limit:

$\tilde{\omega}=\left(\frac{s_{12}}{x}+\frac{s_{24}}{x-1}+\frac{s_{23}}{x-y}\right) d x+\left(\frac{s_{13}}{y}+\frac{s_{34}}{y-1}+\frac{s_{23}}{y-x}\right) d y$, 
where we made an identification of $\alpha$ 's with specific Mandelstam invariants, $s_{a b}=\left(k_{a}+k_{b}\right)^{2}$, involving ingoing lightlike momenta $k_{a}$. Note that it preserves the condition $\sum_{i=1}^{6} \alpha_{i}=0$ due to momentum conservation. Using a pair of twisted cocycles, for example,

$$
\begin{aligned}
& \tilde{\varphi}_{L}=d \log \frac{f_{1}}{f_{5}} \wedge d \log \frac{f_{5}}{f_{4}}=-\frac{d x \wedge d y}{x(x-y)(y-1)}, \\
& \tilde{\varphi}_{R}=d \log \frac{f_{2}}{f_{5}} \wedge d \log \frac{f_{5}}{f_{3}}=\frac{d x \wedge d y}{y(y-x)(x-1)},
\end{aligned}
$$

we can evaluate their intersection number at this singular arrangement via Eq. (7), giving

$$
\left\langle\tilde{\varphi}_{L}, \tilde{\varphi}_{R}\right\rangle_{\tilde{\omega}}=\frac{1}{s_{23}}\left(\frac{1}{s_{12}+s_{13}+s_{23}}+\frac{1}{s_{24}+s_{34}+s_{23}}\right),
$$

which is indeed an example of a biadjoint scalar partial amplitude [33,34]. The limit $\varepsilon \rightarrow 0$ needs to be taken before performing the integration Eq. (7). Evaluating it at finite $\varepsilon$ yields identically zero in agreement with Eq. (6), since $L=$ $\left(f_{1}, f_{5}, f_{4}\right)$ and $R=\left(f_{2}, f_{5}, f_{3}\right)$ have no intersection points in a generic arrangement. In general, intersection numbers change discontinuously depending on the topology, but not geometry, of the arrangement.

With this example we illustrated how the new prescription Eq. (7) provides a way of calculating intersection numbers even at singular hyperplane arrangements, such as the ones giving rise to scattering amplitudes. Let us now flesh out details of this construction in its full generality.

General formula.-In general, let us consider a generic arrangement of $k$ hyperplanes on $\mathbb{C P}^{m}$. They are described with

$$
f_{i}=c_{1 i}+\sum_{a=2}^{m+1} c_{a i} \sigma_{a}
$$

where $\sigma_{a}$ for $a=2,3, \ldots, m+1$ are the inhomogeneous coordinates on $\mathbb{C P}^{m}$. This corresponds to a point in the Grassmannian, $C \in \operatorname{Gr}(m+1, k)$. A given arrangement is nonsingular if all maximal minors of $C$ are nonvanishing. The resulting manifold is $X=\mathbb{C P}^{m} \backslash \cup_{i=1}^{k}\left\{f_{i}=0\right\}$, and the twist 1-form $\omega$ is defined as in Eq. (2), giving

$$
\omega=\sum_{a=2}^{m+1}\left(\sum_{i=1}^{k} \frac{\alpha_{i} c_{a i}}{c_{1 i}+\sum_{b=2}^{m+1} c_{b i} \sigma_{b}}\right) d \sigma_{a},
$$

with $\sum_{i=1}^{k} \alpha_{i}=0$ and $\alpha$ 's sufficiently generic.

On this space we introduce the $m$ th twisted cohomology group [7]:

$$
H^{m}\left(X, \nabla_{\omega}\right)=\left\{\varphi \mid \nabla_{\omega} \varphi=0\right\} /\left\{\nabla_{\omega} \xi\right\},
$$

where $\nabla_{\omega}=d+\omega \wedge$ is the connection and $\xi$ is any smooth $(m-1)$-form on $X$. The dimension of this group is $d=\left(\begin{array}{c}k-2 \\ m\end{array}\right)$. Its elements are called twisted cocycles. One choice of a basis is the one constructed from cocycles of the form

$$
\begin{aligned}
\varphi_{L} & =d \log \frac{f_{L(1)}}{f_{L(2)}} \wedge d \log \frac{f_{L(2)}}{f_{L(3)}} \wedge \cdots \wedge d \log \frac{f_{L(m)}}{f_{L(m+1)}} \\
& =\hat{\varphi}_{L} d \sigma_{2} \wedge d \sigma_{3} \wedge \cdots \wedge d \sigma_{m+1},
\end{aligned}
$$

for example, with $1=L(1)<L(2)<\cdots<L(m+1)<k$ [12]. It is known that an arbitrary twisted cocycle can be expressed in a logarithmic basis [7], such as the one above. Similarly, the dual $m$ th twisted cohomology is defined with the connection $\nabla_{-\omega}$, whose basis can be chosen to be the same as in Eq. (15).

Intersection numbers are normally computed using the definition Eq. (5) with normalization $1 /(2 \pi i)^{m}$ for twisted cocycles $\varphi_{L}$ and $\varphi_{R}$ in the original and dual cohomologies, respectively; see Ref. [13]. In generic arrangements they evaluate, up to an overall sign, to [12]

$$
\left\langle\varphi_{L}, \varphi_{R}\right\rangle_{\omega}= \pm \sum_{\left\{f_{1}, f_{2}, \ldots, f_{m}\right\} \in L, R} \frac{1}{\alpha_{1} \alpha_{2} \cdots \alpha_{m}} .
$$

In singular cases, the above expression requires careful evaluation using blowups; see Ref. [13].

Here, we give an alternative formula as an integral localizing on the zeros of $\omega$ :

$$
\left\langle\varphi_{L}, \varphi_{R}\right\rangle_{\omega}=\frac{1}{(-2 \pi i)^{m}} \oint_{\bigwedge_{a=2}^{m+1}\left\{\left|\omega_{a}\right|=\epsilon\right\}} \frac{\varphi_{L} \hat{\varphi}_{R}}{\prod_{a=2}^{m+1} \omega_{a}} .
$$

The above formula is valid even at singular hyperplane arrangements. We used a more precise notation in terms of a multidimensional residue around the zeros of Eq. (13), in place of $\delta$ functions localizing the integral like in the example Eq. (7).

Proof.-Intersection numbers of twisted cocycles satisfy twisted period relations [6]:

$\left\langle\varphi_{L}, \varphi_{R}\right\rangle_{\omega}=\frac{1}{(2 \pi i)^{m}} \sum_{\alpha, \beta=1}^{d} \int_{\mathcal{A}_{\alpha}} e^{\int \omega} \varphi_{L} \mathbf{H}_{\beta \alpha}^{-1} \int_{\mathcal{B}_{\beta}} e^{-\int \omega} \varphi_{R}$.

By $\exp \int \omega$ we denote the multivalued function $\prod_{i=1}^{k} f_{i}^{\alpha_{i}}$ with some choice of a branch. We have two sets of $d$ twisted cycles $\left\{\mathcal{A}_{\alpha}\right\}$ and $\left\{\mathcal{B}_{\beta}\right\}$ forming bases of their respective homology groups. Here, $\mathbf{H}$ is the intersection matrix, whose entries are the intersection numbers of these cycles [6-8]. Since integrals in the above expression do not generically converge at the same time, they are to be understood in terms of their analytic continuation. In order 
to use localization arguments, however, we need to define appropriate bases of cycles which fix this problem.

Following the Picard-Lefschetz prescription [35], we choose bases of twisted cycles to be the paths of steepest descent and ascent of $\exp \int \omega$ on the same branches, denoted by $\left\{\mathcal{J}_{\alpha}\right\}$ and $\left\{\mathcal{K}_{\beta}\right\}$, respectively. By definition, each of them passes through exactly one critical point $\sigma_{a}^{(\alpha)}$ of $\exp \int \omega$, or equivalently a zero of $\omega$. Therefore, cycles intersect only at these points and the intersection matrix becomes an identity matrix, $\mathbf{H}_{\alpha \beta}=\delta_{\alpha \beta}$, giving

$\left\langle\varphi_{L}, \varphi_{R}\right\rangle_{\omega}=\frac{1}{(2 \pi i)^{m}} \sum_{\alpha=1}^{d} \int_{\mathcal{J}_{\alpha}} e^{\int \omega} \varphi_{L} \int_{\mathcal{K}_{\alpha}} e^{-\int \omega} \varphi_{R}$.

Cycles $\left\{\mathcal{K}_{\alpha}\right\}$ are now paths of steepest descent of $\exp -\int \omega$. Crucially, this means that each integral on the right-hand side of the above equation converges. Let us rescale $\omega \rightarrow \tau \omega$ and take $\tau \rightarrow \infty$. In this limit, each integral localizes on precisely one of the $d$ critical points:

$$
\lim _{\tau \rightarrow \infty}\left\langle\varphi_{L}, \varphi_{R}\right\rangle_{\tau \omega}=\left.\frac{1}{(-\tau)^{m}} \sum_{\alpha=1}^{d} \operatorname{det}^{-1}\left[\frac{\partial^{2} \int \omega}{\partial \sigma_{a} \partial \sigma_{b}}\right] \hat{\varphi}_{L} \hat{\varphi}_{R}\right|_{\sigma_{a}=\sigma_{a}^{(\alpha)}},
$$

where the exponential factors cancel out between the two integrals for each critical point, and $\partial \int \omega / \partial \sigma_{a}=\omega_{a}$ is single valued. Since the number of critical points equals the dimension of the homology group $d$ [7], all zeros of $\omega$ are counted.

On the other hand, intersection numbers $\left\langle\varphi_{L}, \varphi_{R}\right\rangle_{\tau \omega}$ are known to scale homogeneously as $\tau^{-m}$, provided that $\varphi_{L}$ and $\varphi_{R}$ are expressed in a logarithmic basis [12], cf. Eq. (16). We therefore conclude that the above localization formula is exact in $\tau$ and hence we can set $\tau=1$. Expressing the result in terms of a multidimensional residue, this proves our claim Eq. (17).

Scattering amplitudes as intersection numbers.-Let us now consider a special case in which the arrangement of hyperplanes produces the moduli space of $n$-punctured Riemann spheres, $X=\mathcal{M}_{0, n}$. The dimension of $X$ is $m=n-3$, and $k=n(n-3) / 2+1$ hyperplanes are given by

$$
\bigcup_{a=2}^{n-2}\left\{\sigma_{a}=0\right\} \bigcup_{a=2}^{n-2}\left\{\sigma_{a}-1=0\right\} \bigcup_{2 \leq a<b \leq n-2}\left\{\sigma_{a}-\sigma_{b}=0\right\},
$$

with the last one located at infinity. We introduce three coordinates $\left(\sigma_{1}, \sigma_{n-1}, \sigma_{n}\right)=(0,1, \infty)$ and choose the coefficients $\alpha$ for hyperplanes $\left\{\sigma_{a}-\sigma_{b}=0\right\}$ to be Mandelstam invariants $s_{a b}$. For massless kinematics they add up to zero by momentum conservation. There exists a special kinematic region with all $s_{a b}$ except for $s_{1, n-1}$ being positive [36], where all $(n-3)$ ! zeros of $\omega$ lie in distinct chambers in the real section of the moduli space [37].
The dimension of the cohomology group $d$ undergoes a huge reduction compared to a generic arrangement, from $\left(\begin{array}{c}n(n-3) / 2-1 \\ n-3\end{array}\right)$ to $(n-3)$ ! in this singular limit. It also gains an enhanced $\operatorname{SL}(2, \mathbb{C})$ redundancy, $\sigma_{a} \rightarrow\left(A \sigma_{a}+B\right) /\left(C \sigma_{a}+D\right)$, with $A D-B C=1$.

A basis of twisted cocycles can be written using ParkeTaylor forms [10] for the $(n-3)$ ! permutations $\alpha$ :

$$
\begin{aligned}
\operatorname{PT}(\alpha) & =d \log \frac{\sigma_{1, \alpha(2)}}{\sigma_{\alpha(2), \alpha(3)}} \wedge \cdots \wedge d \log \frac{\sigma_{\alpha(n-3), \alpha(n-2)}}{\sigma_{\alpha(n-2), n-1}} \\
& =(-1)^{n} \frac{d \sigma_{\alpha(2)} \wedge d \sigma_{\alpha(3)} \wedge \cdots \wedge d \sigma_{\alpha(n-2)}}{\sigma_{1, \alpha(2)} \sigma_{\alpha(2), \alpha(3)} \cdots \sigma_{\alpha(n-2), n-1}},
\end{aligned}
$$

where $\sigma_{a b}=\sigma_{a}-\sigma_{b}$. The twist 1-form $\omega$ becomes a linear combination of scattering equations [38], $E_{a}$ :

$$
\omega=\sum_{a=2}^{n-2}\left(\sum_{\substack{b=1 \\ b \neq a}}^{n} \frac{s_{a b}}{\sigma_{a b}}\right) d \sigma_{a}=\sum_{a=2}^{n-2} E_{a} d \sigma_{a} .
$$

With these assignments, intersection numbers Eq. (17) become scattering amplitudes in the CHY formulation $[32,33,39]$. Physically, the twist 1-form Eq. (21) translates between singularities of the $S$ matrix and boundaries of the moduli space. Quantum field theory whose amplitudes are being computed depends on the choice of $\varphi_{L}$ and $\varphi_{R}$. For instance, the ingredient $P f^{\prime} \Psi$ defined in Ref. [32] can be expanded in the basis of twisted cocycles, and the pairings

$$
\left\langle P f^{\prime} \Psi, P f^{\prime} \Psi\right\rangle_{\omega}, \quad\left\langle\mathrm{PT}(\alpha), P f^{\prime} \Psi\right\rangle_{\omega}, \quad\langle\mathrm{PT}(\alpha), \operatorname{PT}(\beta)\rangle_{\omega}
$$

give amplitudes of Einstein gravity, Yang-Mills theory, and biadjoint scalar, respectively.

In this case, Eq. (18) reduces to the so-called chiral KLT relation $[9,40,41]$, and $\tau$, which is a rescaling parameter of the Mandelstam invariants, can be identified with the inverse string tension $\alpha^{\prime}$. In particular, this proves the following two statements.

(i) The result of chiral KLT is a field-theory scattering amplitude in the CHY prescription. This provides mathematical foundations for more physical considerations coming from string theory [42-47].

(ii) Since the $\alpha^{\prime} \rightarrow 0$ limit of a closed string amplitude is unaffected up to a sign by the $\alpha^{\prime} \rightarrow-\alpha^{\prime}$ replacement on one side of the KLT relation, the field-theory limit of a closed string amplitude is given by the CHY formula.

Recall that both $\varphi_{L}$ and $\varphi_{R}$ are elements of the twisted cohomology groups, and, in particular, are required to have only logarithmic singularities on the boundaries of the moduli space. Similarly, Mandelstam invariants $s_{a b}$ entering $\omega$ are required to add up to zero, making the above results valid only for massless external states.

Outlook.-Let us put the results of this Letter into a broader perspective. In Ref. [10] we found that twisted 
cycles and cocycles associated to the moduli space $\mathcal{M}_{0, n}$ play a special role in scattering amplitudes. Three types of pairings calculate the following classes of amplitudes:

\begin{tabular}{ll}
\hline Pairing & \multicolumn{1}{c}{ Class } \\
\hline$\langle$ cocycle, cocycle $\rangle$ & Closed string, CHY \\
{$[$ cycle, cocycle $\rangle$} & Open string \\
{$[$ cycle, cycle $]$} & Inverse KLT kernel \\
\hline
\end{tabular}

In this Letter we studied intersection numbers of twisted cocycles, which fall into the first class. Within it, the difference between closed string and CHY-type amplitudes comes from a different choice of the dual cohomology group; see Ref. [10] and references therein.

Every twisted cocycle has a corresponding cycle, whose boundaries coincide with logarithmic singularities of the former. For instance, Parke-Taylor forms [Eq. (20)] map to associahedra tiling the moduli space $[10,48]$. Intersection numbers of both cycles and cocycles can then be described using adjacency properties of the associahedra [10,49], or their linear combinations [50-53].

One of the advantages of this way of thinking is that it allows for geometric understanding of relations between different amplitudes, in particular the KLT relations $[9,10]$. They can be summarized using convenient bra-ket notation; see Ref. [13].

It is natural to expect that similar interpretation in terms of intersection numbers can be made at higher loops or for specific theories in four dimensions, especially given recent evidence that field-theory loop integrands can be obtained from genus-zero Riemann surfaces [54,55] and obey KLT formulas [56]. The additional challenge is to consider nongeneric kinematics on top of singular hyperplane arrangements.

We thank Freddy Cachazo, Job Feldbrugge, Alfredo Guevara, Song He, Julio Parra Martínez, Oliver Schlotterer, Piotr Tourkine, and Masaaki Yoshida for useful comments and discussions. This research was supported in part by Perimeter Institute for Theoretical Physics. Research at Perimeter Institute is supported by the Government of Canada through the Department of Innovation, Science and Economic Development Canada and by the Province of Ontario through the Ministry of Research, Innovation and Science.

\section{*smizera@pitp.ca}

[1] E. Witten, Perturbative gauge theory as a string theory in twistor space, Commun. Math. Phys. 252, 189 (2004).

[2] N. Arkani-Hamed, J. L. Bourjaily, F. Cachazo, A. B. Goncharov, A. Postnikov, and J. Trnka, Scattering Amplitudes and the Positive Grassmannian (Cambridge University Press, Cambridge, England, 2016).

[3] N. Arkani-Hamed and J. Trnka, The amplituhedron, J. High Energy Phys. 10 (2014) 030.
[4] M. Atiyah, M. Dunajski, and L. Mason, Twistor theory at fifty: from contour integrals to twistor strings, Proc. R. Soc. A 473, 20170530 (2017).

[5] D. Eisenbud and J. Harris, 3264 and All That: A Second Course in Algebraic Geometry (Cambridge University Press, Cambridge, England, 2016).

[6] K. Cho and K. Matsumoto, Intersection theory for twisted cohomologies and twisted Riemann's period relations I, Nagoya mathematical Journal 139, 67 (1995).

[7] K. Aomoto and M. Kita, Theory of Hypergeometric Functions, Springer Monographs in Mathematics (Springer, Tokyo, 2011).

[8] M. Yoshida, Hypergeometric Functions, My Love: Modular Interpretations of Configuration Spaces, Aspects of Mathematics (Vieweg+Teubner Verlag, Braunschweig/ Wiesbaden, 2013).

[9] H. Kawai, D. C. Lewellen, and S. H. H. Tye, A relation between tree amplitudes of closed and open strings, Nucl. Phys. B269, 1 (1986).

[10] S. Mizera, Combinatorics and topology of Kawai-LewellenTye relations, J. High Energy Phys. 08 (2017) 097.

[11] Intersection numbers are normally defined for cycles. It is conventional [6-8] to use the same name for a pairing of cocycles, even though its geometrical interpretation is different. For twisted cohomologies [7], intersection numbers are in general not integers.

[12] K. Matsumoto, Intersection numbers for logarithmic kforms, Osaka Journal of mathematics 35, 873 (1998).

[13] See Supplemental Material at http://link.aps.org/ supplemental/10.1103/PhysRevLett.120.141602 for a brief review of the conventional way of calculating intersection numbers of twisted cocycles, as well as a description of the bra-ket notation for twisted cycles and cocycles, which includes Refs. [14-31].

[14] P. Deligne and G. Mostow, Monodromy of hypergeometric functions and non-lattice integral monodromy, Publ. Math. Inst. Hautes Étud. Sci. 63, 5 (1986).

[15] K. Matsumoto, Intersection numbers for 1-forms associated with confluent hypergeometric functions, Funkcialaj ekvacioj Serio internacia 41, 291 (1998).

[16] K. Ohara, Intersection numbers of twisted cohomology groups associated with Selberg-type integrals (1998), http:// www.math.kobe-u.ac.jp/HOME/ohara/Math/980523.ps.

[17] K. Mimachi and M. Yoshida, Intersection numbers of twisted cycles and the correlation functions of the conformal field theory, Commun. Math. Phys. 234, 339 (2003).

[18] K. Mimachi and M. Yoshida, Intersection numbers of twisted cycles associated with the selberg integral and an application to the conformal field theory, Commun. Math. Phys. 250, 23 (2004).

[19] N. E. J. Bjerrum-Bohr, P. H. Damgaard, B. Feng, and T. Sondergaard, Gravity and Yang-Mills amplitude relations, Phys. Rev. D 82, 107702 (2010).

[20] N. E. J. Bjerrum-Bohr, P. H. Damgaard, B. Feng, and T. Sondergaard, Proof of gravity and Yang-Mills amplitude relations, J. High Energy Phys. 09 (2010) 067.

[21] N. E. J. Bjerrum-Bohr, P. H. Damgaard, T. Sondergaard, and P. Vanhove, The momentum kernel of gauge and gravity theories, J. High Energy Phys. 01 (2011) 001. 
[22] C. R. Mafra, O. Schlotterer, and S. Stieberger, Complete N-point superstring disk amplitude I. Pure spinor computation, Nucl. Phys. B873, 419 (2013).

[23] K. Ohmori, Worldsheet geometries of ambitwistor string, J. High Energy Phys. 06 (2015) 075.

[24] S. Mizera and G. Zhang, String-theoretic deformation of the Parke-Taylor factor, Phys. Rev. D 96, 066016 (2017).

[25] G. Domokos, S. Kovesi-Domokos, and E. Schonberg, Fully reggeized scattering amplitudes, Phys. Rev. D 2, 1026 (1970).

[26] I. Bars and F. Gürsey, Duality and the lorentz group, Phys. Rev. D 4, 1769 (1971).

[27] I. Montvay, Multilinear lorentz-invariant functionals at the physical points, Phys. Rev. D 3, 2532 (1971).

[28] L. Brink and A. Kihlberg, Dual models with SL(2,C) symmetry, Nucl. Phys. B46, 505 (1972).

[29] I. O. Moen, Lorentz invariance and duality, Nuovo Cimento Soc. Ital. Fis. 10A, 784 (1972).

[30] R. Musto, F. Nicodemi, M. L. Paciello, and B. Taglienti, Fully-symmetric dual models and Lorentz invariance, Nuovo Cimento Soc. Ital. Fis. 7A, 407 (1972).

[31] M. L. Paciello, A. Sciarrino, and B. Taglienti, Projective invariance of dual-resonance models from spin analyticity and lorentz invariance, Nuovo Cimento Soc. Ital. Fis. 14A, 591 (1973).

[32] F. Cachazo, S. He, and E. Y. Yuan, Scattering of Massless Particles in Arbitrary Dimensions, Phys. Rev. Lett. 113, 171601 (2014).

[33] F. Cachazo, S. He, and E. Y. Yuan, Scattering of massless particles: scalars, gluons and gravitons, J. High Energy Phys. 07 (2014) 033.

[34] N. E. J. Bjerrum-Bohr, P. H. Damgaard, R. Monteiro, and D. O'Connell, Algebras for amplitudes, J. High Energy Phys. 06 (2012) 061.

[35] V. Arnold, A. Varchenko, and S. Gusein-Zade, Singularities of Differentiable Maps: Volume II Monodromy and Asymptotic Integrals, Monographs in Mathematics (Birkhäuser Boston, 2012).

[36] F. Cachazo, S. Mizera, and G. Zhang, Scattering equations: real solutions and particles on a line, J. High Energy Phys. 03 (2017) 151.

[37] It appears to be true in general that for $\alpha_{i}>0, i=1$, $2, \ldots, k-1$, all zeros of $\omega$ are placed in distinct chambers of $\operatorname{Re}(X)$ not bounded by $\left\{f_{k}=0\right\}$.

[38] F. Cachazo, S. He, and E. Y. Yuan, Scattering equations and Kawai-Lewellen-Tye orthogonality, Phys. Rev. D 90, 065001 (2014).
[39] L. Dolan and P. Goddard, Proof of the formula of Cachazo, $\mathrm{He}$ and Yuan for Yang-Mills tree amplitudes in arbitrary dimension, J. High Energy Phys. 05 (2014) 010.

[40] Y.-t. Huang, W. Siegel, and E. Y. Yuan, Factorization of chiral string amplitudes, J. High Energy Phys. 09 (2016) 101.

[41] M. M. Leite and W. Siegel, Chiral closed strings: four massless states scattering amplitude, J. High Energy Phys. 01 (2017) 057.

[42] W. Siegel, Amplitudes for left-handed strings, arXiv: 1512.02569.

[43] E. Casali and P. Tourkine, On the null origin of the ambitwistor string, J. High Energy Phys. 11 (2016) 036.

[44] K. Lee, S.-J. Rey, and J. A. Rosabal, A string theory which isn't about strings, J. High Energy Phys. 11 (2017) 172.

[45] T. Azevedo and R. L. Jusinskas, Connecting the ambitwistor and the sectorized heterotic strings, J. High Energy Phys. 10 (2017) 216.

[46] Y. Li and W. Siegel, Chiral superstring and CHY amplitude, arXiv:1702.07332.

[47] E. Casali and P. Tourkine, Windings of twisted strings, Phys. Rev. D 97, 061902 (2018).

[48] S. L. Devadoss, Homotopy invariant algebraic structures: A conference in honor of J. Michael Boardman, Contemp. Math. 239, 91 (1999).

[49] S. Mizera, Inverse of the string theory KLT kernel, J. High Energy Phys. 06 (2017) 084.

[50] M. Carr and S. L. Devadoss, Coxeter complexes and graphassociahedra, Topol. Appl. 153, 2155 (2006).

[51] A. Postnikov, Permutohedra, associahedra, and beyond, Int. Math. Res. Not. 2009, 1026 (2009).

[52] X. Gao, S. He, and Y. Zhang, Labelled tree graphs, Feynman diagrams and disk integrals, J. High Energy Phys. 11 (2017) 144.

[53] N. Early, Generalized permutohedra, scattering amplitudes, and a cubic three-fold, arXiv:1709.03686.

[54] Y. Geyer, L. Mason, R. Monteiro, and P. Tourkine, Loop Integrands for Scattering Amplitudes from the Riemann Sphere, Phys. Rev. Lett. 115, 121603 (2015).

[55] Y. Geyer, L. Mason, R. Monteiro, and P. Tourkine, Two-loop scattering amplitudes from the Riemann sphere, Phys. Rev. D 94, 125029 (2016).

[56] S. He and O. Schlotterer, New Relations for Gauge-Theory and Gravity Amplitudes at Loop Level, Phys. Rev. Lett. 118, 161601 (2017). 